\title{
Circulating Cell Free DNA and Citrullinated Histone H3 As Useful Biomarkers of NETosis In Endometrial Cancer.
}

\section{Livia Ronchetti}

IRE: Istituto Regina Elena

Irene Terrenato

IRE: Istituto Regina Elena

Giacomo Corrado

Policlinico A Gemelli: Policlinico Universitario Agostino Gemelli

\section{Frauke Goeman}

IRE: Istituto Regina Elena

\section{Sara Donzelli}

IRE: Istituto Regina Elena

\section{Chiara Mandoj}

IRE: Istituto Regina Elena

\section{Roberta Merola}

Istituto Regina Elena

\section{Ashanti Zampa}

IRE: Istituto Regina Elena

\section{Mariantonia Carosi}

ire regina elena

\section{Giovanni Blandino}

IRE: Istituto Regina Elena

\section{Laura Conti}

IRE: Istituto Regina Elena

\section{Anna Maria Lobascio}

IRE: Istituto Regina Elena

Marcello lacobelli

IRE: Istituto Regina Elena

\section{Enrico Vizza}

IRE: Istituto Regina Elena

\section{Giulia Piaggio}

IRE: Istituto Regina Elena

Aymone Gurtner ( $\square$ aymone.gurtner@ifo.gov.it )

National Cancer Institute: Istituto Regina Elena https://orcid.org/0000-0002-7661-9059 


\section{Research Article}

Keywords: NETs, NETosis, circulating cell-free DNA, citrullinated Histone H3, Neutrophil Elastase, Tumor induced systemic effects, DNA size distribution, liquid biopsy, DNA cfDNA fragmentation pattern

Posted Date: October 26th, 2021

DOI: https://doi.org/10.21203/rs.3.rs-998151/v1

License: (a) This work is licensed under a Creative Commons Attribution 4.0 International License. Read Full License 


\section{Abstract}

Background. Cancer mortality is mainly caused by organ failure and thrombotic events. It has been demonstrated that NETosis, a chromatin release mechanism implemented by neutrophils, may contribute to these lethal systemic effects. Our aim was to investigate NETosis biomarkers in endometrial cancer (EC).

Methods. The experiments were conducted on 21 healthy subject (HS) with no gynecological conditions, and on 63 EC patients. To assess the presence of NETosis features IHC and IF was performed using antibodies against citrullinated histone H3 (citH3), neutrophil elastase (NE) and H2B. Serum levels of cell free DNA (cfDNA), cell free mitochondrial DNA (cfmtDNA) and citH3 were measured by qPCR using one microliter of deactivated serum, and by ELISA assay respectively. Fragmentation pattern of serum cfDNA was analyzed using the Agilent 2100 Bioanalyzer and High Sensitivity DNA Chips. Receiver operating characteristic (ROC) analysis was used to identify a cut off for cfDNA and cfmtDNA values able to discriminate between ECs and HSs. Correlation analysis and multiple correspondance analysis (MCA), between cfDNA, mitcfDNA, citH3 and blood parameters were used to identify the potential association among serum parameters in EC grades.

Results. We demonstrated the presence of NETosis features in tissues from all EC grades. Serum cfDNA and cfmtDNA levels discriminate ECs from HSs and a direct correlation between citH3 and cfDNA content and an inverse correlation between cfmtDNA and citH3 in EC sera was observed, not detectable in HSs. MCA indicates cfDNA, cfmtDNA and citH3 as features associated to $\mathrm{G} 1$ and $\mathrm{G} 2$ grades. A correlation between increased levels of cfDNA, citH3 and inflammation features was found. Finally, serum nucleosomal cfDNA fragmentation pattern varies in EC sera and correlates with increased levels of cfDNA, citH3, lymphocytes and fibrinogen.

Conclusion. Our data highlight the occurrence of NETosis in EC and indicate serum cfDNA and citH3 as noninvasive biomarkers of tumor-induced systemic effects in endometrial cancer.

\section{Background}

Tumor-induced systemic effects, such as thrombosis, organ failure and metastasis, are the cause of nearly all cancer-related mortalities. It has been demonstrated that the rapid DNA release mechanisms of activated immune-competent cells contribute to these systemic effects $(1,2,3,4)$. During immune responses, neutrophils are among the first cells to reach the site of inflammation. The increased number of tumor associated neutrophils is linked to poor outcomes in different type of cancers, and many patients with advanced cancer show high levels of blood neutrophils $(5,6,7)$. It has been shown that various tumors are capable to predispose circulating neutrophils to produce neutrophil extracellular traps (NETs) causing systemic thrombosis and thromboembolism which are often associated with human cancers, through biological process called NETosis $(4,8,9,10,11,12,13,14)$. NETs are composed of nuclear DNA in a web-like structures extruded from neutrophils

and mixed with granular and some cytoplasmic constituents, such as neutrophil elastase (NE), myeloperoxidase (MPO), and citrullinated histone H3 (citH3), in response to infection or cancer burden (15). Recently, several studies have unveiled unexpected functions of NETosis in promoting tumor development including tumor growth, metastasis and angiogenesis and in protecting tumor cells from cytotoxic immune cells $(16,17,18,19$, $20,12,21,22)$. These evidences suggest that NETosis may provide a novel tool, potentially targetable, for cancer treatment $(23,24,25,14)$. Moreover, several studies indicate that the mechanism of NETosis can provide an explanation for the elevated circulating cell-free DNA (cfDNA) release in blood stream in pathologic conditions 
$(26,27)$ and strongly suggest that these NET-associated molecules are potential tumour biomarkers $(5,28,29$, 30).

We have recently demonstrated the potential of cfDNA as a simple and inexpensive biomarker in endometrial cancer (EC), particularly related to lymphovascular space invasion (LVSI) status $(5,28)$. Despite EC generally has a good prognosis, about a quarter of patients present an unfavorable prognosis due to the advanced stage of disease at the time of diagnosis. Therefore, more efforts are needed to discover new biomarkers useful for early diagnosis and prognosis.

In the present study, a retrospective analysis was conducted on tissues and liquid biopsies to evaluate the presence of NETosis features in EC and they potentially usefulness for diagnosis and/or prognosis. We investigated preoperative cfDNA quantitative and qualitative content, serum levels of citH3 and systemic inflammatory indicators such as fibrinogen, neutrophils, platelets and lymphocytes. We evaluated the prognostic significance of the combination of these factors in EC providing evidences on the possible involvement of NETosis formation in tumor-induced systemic effects.

\section{Methods}

2.1 Patient cohort: All healthy volunteers and EC patients were recruited at the Regina Elena National Cancer Institute. We collected serum samples from 21 healthy volunteers with no gynecological conditions, and 63 EC patients. Patients included in this study were not previously selected, but randomly chosen and recruited between 2014 and 2017. According with the histologic grade, we analyzed samples from 9 G1, 33 G2 and 21 G3 ECs. Supplementary table 1 depicts clinical-pathological characteristics of patients enrolled in this study.

2.2 Immunohistochemistry: Citrullinated Histone H3 expression was evaluated by immunohistochemistry (IHC) on formalin-fixed paraffin-embedded (FFPE) tissues using rabbit polyclonal anti-Histone H3 (citrulline R2 + R8 + R17) (Abcam) at the diluition of 1:50 (citrate based pH 6.0 epitope retrieval solution). Immunoreactions were revealed by a streptavidin-biotin enhanced immunoperoxidase technique in an automated autostainer (Bond III, Leica Biosystem, Milan, Italy), according to the manufacturer's instructions. Immunoreactivity was evaluated by two investigators (LR and MC) and discordant cases were subsequently discussed and agreed upon.

2.3 Immunofluorescence: Immunofluorescence staining to observe the expression of NETosis markers was performed on formalin-fixed paraffin-embedded (FFPE) tissues utilizing the following primary antibodies: rabbit polyclonal anti-Elane (Atlas) at the diluition of 1:50 (citrate based pH 9.0 epitope retrieval solution); chicken polyclonal anti-Histone H2B (Abcam) at the diluition of 1:500 (citrate based pH 9.0 epitope retrieval solution) rabbit polyclonal anti-Histone H3 (citrulline R2 + R8 + R17) (Abcam) at the diluition of 1:50 (citrate based pH 6.0 epitope retrieval solution). The following were used as secondary antibodies: $\mathrm{Cy}^{\mathrm{TM}} 2$ Affinipure Donkey Antirabbit (Jackson Immuno Research) at the diluition of 1:400, $\mathrm{CY}^{\mathrm{TM}} 3$ Affinipure Donkey Anti-chicken (Jackson Immuno Research) at the diluition of 1:400. The colorations were read and interpreted by using a fluorescence confocal microscope. Immunoreactivity was evaluated by two investigators (LR and AG) and discordant cases were subsequently discussed and agreed upon. Thermo Scientific DAPI Nuclear Counterstains was used for nuclear staining. 
2.4 Blood processing: Venous blood of cancer patients were obtained before surgery and before the beginning of any treatment. The blood tests were obtained within 24 hours for all patients as routine clinical practice before surgery. NLR, MLR and PLR were defined as the absolute neutrophil count in peripheral blood divided by the absolute lymphocyte count, the absolute count of monocyte divided by the absolute lymphocyte count, and the absolute platelet count in peripheral blood divided by the absolute lymphocyte count, respectively. For serum processing, blood samples were collected in Vacutainer tubes without anticoagulant and processed within 1-4 h. After collection the blood was allowed to clot at room temperature. The blood serum was separated by centrifugation at $1000-2000 \times \mathrm{g}$ for $10 \mathrm{~min}$ in a refrigerated centrifuge and stored at $-80^{\circ} \mathrm{C}$.

2.5 Serum deactivation: $20 \mu \mathrm{L}$ of each serum sample were mixed with $20 \mu \mathrm{L}$ of a preparation buffer $(2.5 \%$ of tween $20,50 \mathrm{mmol} / \mathrm{L} \mathrm{Tris-HCl}$, and $1 \mathrm{mmol} / \mathrm{L}$ EDTA). This mixture was digested with proteinase $\mathrm{K}(20 \mu \mathrm{g})$ solution for $50 \mathrm{~min}$ (Promega) at $56^{\circ} \mathrm{C}$, followed by $5 \mathrm{~min}$ of heat deactivation and insolubilization for $10 \mathrm{~min}$ at $95^{\circ} \mathrm{C}$. After subsequent centrifugation of $10,000 \times \mathrm{g}$ for $5 \mathrm{~min}$, supernatant was used as a template for each quantitative real-time polymerase chain reaction (qRT-PCR) using SYBR Green Power Up Master Mix (Thermo Scientific) followed by evaluation of the average of CT values from triplicate reactions from Real Time PCR software.

2.6 DNA extraction: Isolation from $1 \mathrm{ml}$ of blood serum was performed with commercial kit for blood DNA according to manufacturer's specifications from Qiamp Circulating Nucleid Acid kit (QIAGEN). Concentration and purity of the extracted DNA were evaluated by performing a Qubit Fluorometric Quantification.

2.7 Bioanalyzer analysis: DNA was isolated from $1 \mathrm{ml}$ of blood serum with commercial kit for blood DNA according to isolation manufacturer's specifications from Qiamp Circulating Nucleid Acid kit (QIAGEN). Microfluidic electrophoresis using the Agilent 2100 Bioanalyzer and High Sensitivity DNA Chips (Agilent technologies Inc., Palo Alto, CA, USA) was performed to assess DNA fragment length for a size range between 35 and 10.380 base pairs (bp) based on manufacturer's recommended protocol. Bioanalyzer 2100 Expert Software was used for the analysis of electrophoretic runs.

2.8 Measurement of cfDNA levels: SYBR Gold Nucleic Acid Gel Stain (Invitrogen) was diluted first at 1:1000 in dimethyl sulphoxide (DMSO) and then at 1:8 in phosphate-buffered saline (PBS). Ten microliters of DNA solutions or sera were applied to a black 96 -well plates. Forty microliters of diluted SYBR Gold were added to each well (final dilution 1:10,000) and fluorescence measured with a 96well fluorometer at an emission wavelength of $535 \mathrm{~nm}$ and an excitation wavelength of $485 \mathrm{~nm}$. Serum samples were diluted in PBS fivefold (20\%). Assay was performed in triplicate. Standards were prepared with commercial Salmon sperm DNA.

2.9 qPCR: One microliter of deactivated serum was used and both the mtDNA and nDNA content were measured by qPCR using SYBR Green Power Up Master Mix (Applied Biosystems, CA, USA) followed by evaluation of the average $C T$ values from triplicate reactions from Real Time PCR software. Primers were designed and used for relative quantification for mtDNA to $\mathrm{nDNA}$ content. Two primer pairs were used for the amplification of two mitochondrial genes: MT-ND1 and mtDNA 16S. One primer set was used for the amplification of the single-copy nuclear gene 36B4. Primers sequences will be: forward ND1: 5'-CCCTAAAACCCGCCACATCT-3' and reverse ND1: 5'-GAGCGATGGTGAGAGCTAAGGT-3'; forward mtDNA 16S 5'-CAGCCGCTATTAAAGGTTCG-3' and reverse: mtDNA 16S 5'-CCTGGATTACTCCGGTCTGA-3'; forward 36B4 5'-CAGCAAGTGGGAAGGTGTAATCC-3' and reverse: 36B4 5'- 
CCCATTCTATCATCAACGGGTACAA-3'. To determine the mtDNA content relative to nDNA, the following equations were used: $\triangle \mathrm{CT}=(\mathrm{nDNA} C \mathrm{CT}-\mathrm{mtDNACT})$. Relative mitochondrial DNA content $=2 \times 2 \Delta \mathrm{CT}$.

2.10 ELISA assay: To quantify CitH3 in the blood, we employed the citrullinated Histone H3 (Clone 11D3) ELISA Kit (Cayman) following the manufacturer's recommended protocol.

2.11 Statistics: The examined variables were not normally distributed, as verified by the Shapiro-Wilk test, thus the most suitable non-parametric test was applied to perform comparisons between groups. Receiver operating characteristic (ROC) curves and the relative area under the curve (AUC) were calculated for all continuous variables of interest. Youden's index was used to maximize the difference between sensitivity and specificity in order to individuate the optimal cut-off for each parameter. Multiple correspondance analysis (MCA), a descriptive/exploratory technique designed to analyze simple two-way and multi-way table, was used to obtain an overview of the potential association among parameters. P-values $<0.05$ were considered statistically significant. The SPSS (version 21.0) was used for all statistical analyses.

\section{Results}

\subsection{NETosis occurs in all grades of EC.}

To start to assess the presence of NETosis features in EC, we performed IHC using an antibody against citH3 on EC and, as control, healthy subject (HS) tissues. The disease characteristics of the enrolled patients are described in Supplementary Table 1. Representative IHC staining are shown in figure 1A. In all EC grades, the analysis of citH3 staining revealed a variable level of staining ranging from negative to strong positive samples (Figure 1A). Although the presence of this variability, of note, among all samples, the majority of tumors $(81.25 \%)$ presents an infiltrate stained for citH3, with a high IHC score $(+2,+3)$ in $60 \%$ of $\mathrm{G} 1,78,6 \%$ of $\mathrm{G} 2$, and $93,4 \%$ of G3 (Figure 1C). By contrast, the $4 \mathrm{HSs}$ were negative for this staining (Figure 1B). Interestingly, the percentage of leucocytes expressing citH3 increases from G1 to G3 (Figure 1C). Notably, we could detect citH3 positive staining in the nucleus and cytoplasm of neutrophils as well as extracellular filaments resembling NETs (Figure 1D).

To further understand whether the citH3 positivity accounts for NETosis by activated neutrophils, we performed confocal immunofluorescence experiments on $30 \mathrm{EC}$ tissues allowing visualization of both intra- and extracellular localization of NE, histone H2B, DNA and citH3. Representative images of 2 EC tissues (G2 and G3 respectively), show a colocalization of NE and $\mathrm{H} 2 \mathrm{~B}$ (Figures 2A, 2E; colocalizzation in yellow). On the same tissue sections, a colocalization of NE and DAPI in the nuclei and cytoplasms of tumor-infiltrating neutrophils has been also observed (Figures 2B, 2F; colocalizzation in white). 3D image magnifications of triple staining (NE, $\mathrm{H} 2 \mathrm{~B}, \mathrm{DAPI}$ ) on representative selected areas (withe boxes) further highlight the colocalization among the three markers. Of note, extrusions of DNA (blu) and NE (green) from tumor-infiltrating neutrophils are also visible (white arrows) and are associated with the loss of the trilobated nuclear shape (Figures 2C, D, G). citH3 staining on EC tissues from the same patients indicates strong neutrophil activation (Figure $2 \mathrm{H}, 2 \mathrm{M}$ ). Magnification of a representative field highlights colocalization (in white) between citH3 and DNA both in the nucleus, in the cytoplasm and extracellular space (Figures 2I, L, N, O). Tissues Images of staining of single antibodies are shown in Supplementary Figure 1. 
Of note, we identified different stages of NETosis process. We were able to visualize inactivated neutrophils (Figure 2P, panels I, II, III), early stages of the process characterized by cytoplasmic to nucleus NE translocation (Figure 2P panels IV, V, VI), and late stages featured by nuclear delobulation, membrane rupture and nuclear chromatin and NE release, (Figure 2P, panels VII, VIII, IX).

In conclusion, combined IHC and IF analysis demonstrate the presence of NETosis processes in all grade of EC tissues.

\subsection{Serum citH3 levels correlate with increased cfDNA content in EC.}

Serum citH3 levels have been previously demonstrated to be a biomarker of NETosis as well as cfDNA $(26,27)$. We have previously demonstrated an increase of cfDNA and a decrease of cfmtDNA in EC sera compared with sera of HSs (5). Based on this, we thought to investigate the relationship between serum citH3 levels and cfDNA (both total and mitochondrial) in $63 \mathrm{EC}$ and $22 \mathrm{HS}$ sera. According to our previously results, we observed a significant increase of cfDNA and a decrease of cfmtDNA contents in EC samples compared with HSs (Supplementary Figure 2A, B). According to EC grades the two features significantly discriminate $\mathrm{G} 2$ and G3 grades form HSs (Supplementary Figure 2C, D). By receiver operating characteristic (ROC) analysis we identify a cut off for cfDNA and cfmtDNA values able to strongly discriminate between ECs and HSs, Table 1 highlighting a cut off strong predictive capability of cfDNA and cfmtDNA values. Median values of citH3 were, instead, comparable in EC and HS sera (Figure $3 \mathrm{~A}$ ) as well as in different EC grades (Figure 3B). Interestingly, categorizing by ROC cut off cfDNA and cfmtDNA value distributions, we observed that citH3 levels were significantly higher in samples with high values of cfDNA (Figure 3C) and low values of cfmtDNA (Figure 3D). Next, we investigated the correlation between these three features. Results showed a significant, although fair, direct correlation between citH3 and cfDNA as well as an inverse correlation with cfmtDNA (Table 2). No correlation has been found in HSs (data not shown). Analyzing the correlations among histological grades, we found a significant direct correlation between citH3 and cfDNA in G2 samples. Beside, an inverse correlation between citH3 and cfmtDNA, as well as between cfDNA and cfmtDNA, occurs in G1 samples (Table 3). In agreement with these results, MCA analysis locates HSs and ECs in two distinct groups (Figure 3E). High levels of citH3 and cfDNA and low levels of cfmtDNA are located closed to G1 and G2. On the contrary, HS samples are located close to citH3 low, cfDNA low, and cfmtDNA high levels. G3, in this analysis, is not associated with any of these features (Figure 3E). In conclusion our data indicate that elevated serum cfDNA concentrations is associated with citH3, a well-known biomarker of NETosis, in G1 and G2 EC patients, supporting the idea that they could be useful to monitor NETosis processes by non-invasive liquid biopsies thus opening the way for new therapeutic strategies in EC. 
Table 1

ROC analysis. (Status =Healthy subjects vs endometrial cancer patents).

\begin{tabular}{|c|c|c|c|c|c|c|}
\hline & $\begin{array}{l}\text { Area Under the Curve } \\
\text { (AUC) }(95 \% \mathrm{Cl})\end{array}$ & $\begin{array}{l}\mathrm{p}- \\
\text { value }\end{array}$ & Test direction & Sensitivity & Specificity & $\begin{array}{l}\text { Cut- } \\
\text { off }\end{array}$ \\
\hline cfDNA & $0.838(0.76-0.92)$ & $<0.001$ & $\begin{array}{l}\text { Higher values } \\
\text { indicate Cancer }\end{array}$ & 0.67 & 1.00 & 712.3 \\
\hline cfmtDNA & $0.854(0.77-0.94)$ & $<0.001$ & $\begin{array}{l}\text { Lower values } \\
\text { indicate Cancer }\end{array}$ & 0.65 & 0.95 & 16.5 \\
\hline citH3 & $0.583(0.44-0.73)$ & 0.339 & $\begin{array}{l}\text { Higher values } \\
\text { indicate Cancer }\end{array}$ & 0.33 & 0.93 & 9.2 \\
\hline
\end{tabular}

Table 2

Correlation analysis between cfDNA, mitcfDNA, citH3, blood parameters in EC sera.

\begin{tabular}{|c|c|c|c|c|c|c|c|c|c|}
\hline & cfmitDNA & citH3 & $\mathrm{Neu}$ & Neu/Lym & Mono/Lym & Mono & Lym & Platelets & Fibr \\
\hline cfDNA & $-0,083$ &, $439^{\star \star}$ & 0,250 & $-0,014$ & $-0,049$ &, $360^{\star *}$ &, $415^{\star \star}$ & $0,318^{*}$ &, $498^{\star *}$ \\
\hline cfmitDNA & 1,000 &,$- 384^{* *}$ & $-0,111$ & $-0,104$ & $-0,008$ & 0,085 & ,088 & 0,223 & 0,028 \\
\hline citH3 & & 1,000 & 0,184 & $-0,051$ & $-0,094$ & 0,213 &, $297^{*}$ & 0,244 & 0,225 \\
\hline
\end{tabular}

Table 3

Correlation analysis between cfDNA, mitcfDNA, citH3, and blood parameters stratified by EC grade.

\begin{tabular}{|llllllllll|}
\hline Grade 1 & cfmitDNA & citH3 & Neu/Lym & Mono/Lym & Fibr & Platelets & Neu & Lym & Mono \\
\hline cfDNA &,$- 683^{*}$ &, 483 &, 429 &, 500 &, $857^{*}$ &, 143 &, $786^{*}$ &, 464 &, 750 \\
\hline cfmitDNA & 1,000 &,$- 733^{*}$ &,- 071 &, 143 &,- 357 &, 143 &,- 429 &,- 536 &,- 107 \\
\hline citH3 & & 1,000 &,- 071 &,- 393 &, 000 &, 321 &, 429 &, 679 &, 143 \\
\hline Grade 2 & cfmitDNA & citH3 & Neu/Lym & Mono/Lym & Fibr & Platelets & Neu & Lym & Mono \\
\hline cfDNA &,- 087 &, $415^{*}$ &,- 091 &,- 144 &, $529^{* *}$ &, 370 &, 262 &, $453^{*}$ &, 347 \\
\hline cfmitDNA & 1,000 &,- 350 &, 219 &, 132 &, 260 &, 106 &, 069 &,- 042 &,- 073 \\
\hline citH3 & & 1,000 &,- 194 &,- 029 &, 276 &, 258 &, 045 &, 271 &, 249 \\
\hline Grade 3 & cfmitDNA & citH3 & Neu/Lym & Mono/Lym & Fibr & Platelets & Neu & Lym & Mono \\
\hline cfDNA &, 388 &, 333 &,- 135 &,- 167 &, 379 &, 434 &, 069 &, 346 &, 254 \\
\hline cfmitDNA & & 1,000 &, 071 &,- 141 &, 131 &, 226 &, 141 &, 074 &, 053 \\
\hline citH3 & & &, 239 &, 136 &,- 441 &,- 011 &, 079 &,- 089 &, 155 \\
\hline *p<0.005; **p<0.001; Neu= neutrophilis; Mono= Monocites; Lym= Lymphocites; Fibr= Fibrinogen & \\
\hline
\end{tabular}




\subsection{Fragmentation pattern of cfDNA in ECs is associated with citH3 serum levels}

cfDNA often appears as a ladder of fragments of different sizes. Interestingly, some experimental evidence suggests that the nature of the fragments changes in cancer patients having prognostic potential $(31,32,33)$. We have previously demonstrated that cfDNA integrity index, determined by the ratio of qPCR Alu247/qPCRAlu115, provides prognostic value in EC (28). However, it is not known yet which is the fragmentation pattern of released cfDNA derived from NETosis. To start to address this issue, we analyzed purified double strand cfDNA samples of 44 ECs and 14 HSs (Supplementary Table 2), with Agilent DNA High Sensitivity Kit. This kit is developed for sizing and quantifying DNA fragments from 35 to $10.380 \mathrm{bp}$. We found that all HSs, with only one exception, have a cfDNA fragmentation profile with a nucleosomal DNA ladder pattern corresponding to mono(195 bp), di- (390 bp), tri- (585) and poly- (from 585 to 9050 bp) nucleosomes (Supplementary Figure 3). Unlike HSs, EC samples present a more variable patterns. All EC samples, regardless of grades, present the short peak fragment of 195bp usually associated with apoptotic events $(31,32,33)$. Among these, $41 \%$ samples present only this short peak (cf mono nucleosome pattern, cfMNP), while 59\% of them present also an accumulation a poly-nucleosomes from 1000 to $9050 \mathrm{bp}$ (cf total nucleosome patterns cfTNP). Moreover, while the intensity of the HS peaks never exceeds 50 fluorescence units (FU), 30\% of ECs present peaks above 50 FU reaching, in some cases, 500 FU (Supplementary Figure 3). This result is in agreement with increased cfDNA levels observed in ECs (Supplementary Figure 2A).

To understand whether the source of cfDNA in ECs could be due to NETosi processes, we clustered EC samples into two groups, depending on the presence of cfTNP or only cfMNP and we investigated their association with serum citH3, cfDNA and cfmtDNA levels. This analysis demonstrated that the presence of cfTNP was associated with a higher concentration of citH3 and cfDNA and a lower cfmtDNA contents (Figure 4A,B,C). To investigate whether a specific cfDNA region accounts for these associations, we splitted total cfDNA in four regions encompassing different DNA lengths (Figure $5 \mathrm{~A}$ ). We found significant direct correlations between all regions and citH3 with significant values in $\mathrm{G} 1$ and $\mathrm{G} 2$. This result is cancer-specific since any correlation has been found in HSs (Table 4). All together these findings suggest that the poly-nucleosomal DNA ladder pattern may reflect NETosis events in EC samples. Finally, from a quantitative point of view, only region 1 is significantly greater in ECs than in HSs respect to the total cfDNA, with an increasing trend from G1 to G3 grades (Figure $5 B, C)$.

Table 4

Correlation among citH3 levels and concentration of region 1, region 2, region $\mathrm{X}$, region $1-X$ and total cfDNA, in EC stratified by grading.

\begin{tabular}{|c|c|c|c|c|c|c|}
\hline & & Region 1 & Region 2 & Region X & Region 1-X & Total cfDNA \\
\hline G1 & citH3 &, $905^{\star *}$ &, $714^{*}$ &, $929 * *$ &, $929 * *$ &, $905^{\star \star}$ \\
\hline $\mathrm{G} 2$ & citH3 &, $781^{* *}$ &, $684^{\star *}$ & ,708** &, 734 *夫 &, $742^{* *}$ \\
\hline G3 & citH3 &, $637^{\star}$ &, 434 & ,549 & ,687* & ,549 \\
\hline \multicolumn{7}{|c|}{${ }^{*} p<0.005 ;{ }^{* *} p<0.001$} \\
\hline
\end{tabular}




\subsection{Correlation between inflammatory/coagulation features and NETosis.}

To assess the possible network between inflammatory features and NETosis, we measure the relationship between inflammatory cell counts and citH3, cfDNA and cfmitDNA levels. A positive correlation was found between citH3 levels and lymphocyte counts (Table 2) in ECs while cfDNA content positively correlates with monocyte, lymphocyte, platelet counts and fibrinogen levels (Table 2). Next, stratification by EC grades disclosed a consistent and positive relationship of cfDNA with both neutrophils and fibrinogen in G1, and with lymphocytes and fibrinogen in G2 (Table 3). No correlation was found between NET markers and inflammatory features in G3 (Table 3). To evaluate whether these markers are associated with the poly-nucleosomal DNA ladder pattern, we measure levels of the inflammatory markers clustering in the two EC subgroup, cfMNP and cfTNP. The results show an increasing trend of lymphocyte counts and fibrinogen levels in cfTNP (Figure 6).

In conclusion our data suggest that citH3 NETosi biomarker, cfDNA content and its fragmentation pattern may reflect the lymphocyte-mediated inflammatory response and coagulation status in ECs discriminating G3 from $\mathrm{G} 1$ and $\mathrm{G} 2$ grades.

\section{Conclusions}

In the present study, a retrospective analysis was conducted on tissues and liquid biopsies to evaluate the presence of NETosis features in endometrial cancer and they potentially usefulness for diagnosis and/or prognosis. We investigated preoperative cfDNA quantitative and qualitative content, serum levels of citH3 and systemic inflammatory indicators such as fibrinogen, neutrophils, platelets and lymphocytes. We evaluated the prognostic significance of the combination of these factors providing evidences on the possible involvement of NETosis formation in tumor-induced systemic effects.

First, by IF and IHC analysis we found that the majority of tumor tissue samples presents an infiltrate of leucocytes positive for the NETosis biomarker citH3, with an increasing trend from $\mathrm{G} 1$ to $\mathrm{G} 3$ grade. To our knowledge, this is the first time that NET structures are described both in intra-cellular infiltrate and extra-cellular spaces in endometrial cancer specimens. Unexpected, serum citH3 levels do not increase in cancer grades and, consequently, we do not observe an association of citH3 tissue staining with its serum levels. This may be due to different mechanisms of cfDNA clearance in tissues versus sera, and/or to the different timing of collection of tissues and blood samples.

Next, we found that serum cfDNA and cfmtDNA levels are useful to discriminate between endometrial cancer patients and healthy subjects. Interestingly, citH3 levels are significantly higher in sera of endometrial cancer samples presenting cfDNA and cfmtDNA levels higher and lower respectively, to the ROC cut off. Further, MCA analysis discriminate endometrial cancer from healthy subjects, locating G1 and G2 grades far from G3 and close to high level of citH3, cfDNA and low levels of cfmtDNA. In agreement, we highlight positive and inverse correlations between cfDNA and cfmtDNA respectively, and citH3 levels in $\mathrm{G} 1$ and $\mathrm{G} 2$. These results strongly support the idea that cfDNA and cfmtDNA levels are the results, at least in part, of NETosis processes. Moreover, since neutrophils, among the leukocytes, contain very limited numbers of mitochondria compared to other cell types (34), the decrease of serum cfmtDNA content further supports the scenario of a NETosis activation in endometrial cancer tissues. 
In recent years, serum analysis of cfDNA in cancer patients has attracted the attention of researchers and clinicians in numerous fields and has opened promising perspectives for diagnosis and prognosis $(31,32,33)$. Beyond quantification and sequencing, the study of cfDNA fragmentation profile has emerged as a promising field in oncology. In this regards, we have recently demonstrated that evaluation of cfDNA integrity index, calculated as qPCR-Alu247 value / qPCR-Alu115, may represent a complementary tool to help EC stratification (28). Here, we decipher the cfDNA fragmentation profile in endometrial cancer patient sera and its relationship with NETosis. Our results highlight the presence of cfDNA fragments spanning from mono- to poly-nuclosomes, all of which could, in G1 and G2 grades, derive from NETosis processes given the consistent correlation of these fragments with high serum levels of citH3. To our knowledge this is one of the few studies investigating serum cfDNA ladder upper to 1000 bps and its correlation with NETosis in an oncological disease.

Collectively, our data demonstrate that serum cfDNA and cfmtDNA are novel non-invasive biomarkers of NETosis in endometrial cancer and pave the way for their diagnostic and prognostic use in combination with serum citH3 content.

\section{Abbreviations}

cell free DNA (cfDNA)

cell free mitochondrial DNA (cfmtDNA)

cf mono nucleosome pattern (cfMNP)

cf total nucleosome patterns (cfTNP)

citrullinated histone H3 (citH3)

endometrial cancer (EC)

fluorescence units (FU)

formalin-fixed paraffin-embedded (FFPE)

healthy subjects (HSs)

myeloperoxidase (MPO)

multiple correspondence analysis (MCA)

neutrophil extracellular traps (NETs)

neutrophil elastase (NE)

receiver operating characteristic (ROC).

\section{Declarations}


Experimental protocol was approved by the Ethics Committee of the Regina Elena National Cancer Institute (Rome, Italy, ifo_058.IFO_AOO.REGISTRO UFFICIALE.U.0013925.14-12-2017), and performed in accordance with the relevant guidelines and regulations. Written informed consent was obtained from all patients and healthy volunteers. Information about patients was obtained by reviewing their medical charts.

\section{Consent for publication}

Not applicable

\section{Availability of data and materials}

The datasets used and/or analyzed during the current study are available from the corresponding author on reasonable request.

\section{Competing interests}

The authors declare that they have no competing interests.

\section{Funding}

This work was supported in part by funds Ricerca Corrente from Ministry of Health, Italy.

\section{Authors' contributions}

$A G, G P$, and $E V$ conceived and designed the research project. LR performed and conceived the experiments. IT performed the statistical analysis. AG, GP and LR wrote the manuscript. MC, GC, MI and AML performed clinical evaluation of samples. GB, FG and SD collaborated in the experimental settings. CM, RM and LC collected serum samples. AZ collected clinical data.

\section{Acknowledgements}

This work has been founded by IRE Internal Projects to AG and EV, and Lazio Region BTO project to EV

\section{References}

1. Borissoff Jl, Joosen IA, Versteylen MO, Brill A, Fuchs T, Savchenko AS, et al. Elevated levels of circulating DNA and chromatin are independently associated with severe coronary atherosclerosis and a prothrombotic state. Arterioscler Thromb Vasc Biol. 2013 Aug;33(8):2032-40. doi:10.1161/ATVBAHA.113.301627.

2. Yang R, Zou X, Tenhunen J, Tønnessen TI. HMGB1 and Extracellular Histones Significantly Contribute to Systemic Inflammation and Multiple Organ Failure in Acute Liver Failure. Mediators Inflamm.

2017;2017:5928078. doi:10.1155/2017/5928078. Review. Yipp BG, Kubes P. NETosis: how vital is it? Blood. 2013, 122(16):2784-94. doi: 10.1182/blood-2013-04-457671. Review.

3. van Montfoort ML, Stephan F, Lauw MN, Hutten BA, Van Mierlo GJ, Solati S, et al. Circulating nucleosomes and neutrophil activation as risk factors for deep vein thrombosis. Arterioscler Thromb Vasc Biol. 2013;33(1):147-51. doi:10.1161/ATVBAHA.112.300498. 
4. Demers M, Krause DS, Schatzberg D, Martinod K, Voorhees JR, Fuchs TA, et al. Cancers predispose neutrophils to release extracellular DNA traps that contribute to cancer-associated thrombosis. Proc Natl Acad Sci U S A. 2012;109(32):13076-81. doi:10.1073/pnas.1200419109. Epub 2012 Jul 23.

5. Cicchillitti L, Corrado G, De Angeli M, Mancini E, Baiocco E, Patrizi L, et al. Circulating cell-free DNA content as blood based biomarker in endometrial cancer. Oncotarget. 2017;8(70):115230-43. doi:10.18632/oncotarget.23247.

6. Vano YA, Oudard S, By MA, Têtu P, Thibault C, Aboudagga H, et al. Optimal cut-off for neutrophil-tolymphocyte ratio: Fact or Fantasy? A prospective cohort study in metastatic cancer patients. PLoS One. 2018;13(4):e0195042. doi:10.1371/journal.pone.0195042.

7. Ethier JL, Desautels DN, Templeton AJ, Oza A, Amir E, Lheureux S. Is the neutrophil-to-lymphocyte ratio prognostic of survival outcomes in gynecologic cancers? A systematic review and meta-analysis. Gynecol Oncol. 2017;145(3):584-94. doi:10.1016/j.ygyno.2017.02.026. Review.

8. Olsson AK, Cedervall J. NETosis in Cancer - Platelet-Neutrophil Crosstalk Promotes Tumor-Associated Pathology. Front Immunol. 2016;7:373. Review.

9. Demers M, Wagner DD. Neutrophil extracellular traps: A new link to cancer-associated thrombosis and potential implications for tumor progression. Oncoimmunology. 2013;2(2):e22946.

10. Guglietta S, Chiavelli A, Zagato E, Krieg C, Gandini S, Ravenda PS, et al. Coagulation induced by C3aRdependent NETosis drives protumorigenic neutrophils during small intestinal tumorigenesis. Nat Commun. 2016;7:11037. doi:10.1038/ncomms11037.

11. Mauracher L-M, Posch F, Martinod K, Grilz E, Däullary T, Hell L, Brostjan C, Zielinski C, Ay C, Wagner DD, Pabinger I, Thaler J. Citrullinated histone H3, a biomarker of neutrophil extracellular trap formation, predicts the risk of venous thromboembolism in cancer patients. J Thromb Haemost. 2018;16:508-18.

12. Thalin C, Lundström S, Seignez C, Daleskog M, Lundström A, Henriksson P, et al. Citrullinated histone H3 as a novel prognostic blood marker in patients with advanced cancer. PLoS One. 2018;13(1):e0191231. doi:10.1371/journal.pone.0191231.

13. Grilz E. Mauracher LM1, Posch F2, Königsbrügge 01, Zöchbauer-Müller S3, Marosi C3, Lang 14, Pabinger I1, Ay C1. Citrullinated histone H3, a biomarker for neutrophil extracellular trap formation, predicts the risk of mortality in patients with cancer. Br J Haematol. 2019 Apr 9. doi:10.1111/bjh.15906.

14. Ronchetti L, Boubaker NS, Barba M, Vici P, Gurtner A, Piaggio G. Neutrophil extracellular traps in cancer: not only catching microbes. J Exp Clin Cancer Res. 2021 Jul 14;40(1):231. doi: 10.1186/s13046-021-02036-z.

15. Boeltz S, Amini P, Anders HJ, Andrade F, Bilyy R, Chatfield S, et al. To NET or not to NET:current opinions and state of the science regarding the formation of neutrophil extracellular traps. Cell Death Differ. 2019 Mar;26(3):395-408. doi:10.1038/s41418-018-0261-x. Epub 2019 Jan 8. Review. PubMed PMID: 30622307; PubMed Central PMCID: PMC6370810.

16. Kessenbrock K, Plaks V, Werb Z. Matrix metalloproteinases: regulators of the tumor microenvironment. Cell. 2010;141(1):52-67. doi:10.1016/j.cell.2010.03.015. Review.

17. Cools-Lartigue J, Spicer J, McDonald B, Gowing S, Chow S, Giannias B, Bourdeau F, Kubes P, Ferri L. Neutrophil extracellular traps sequester circulating tumor cells and promote metastasis. J Clin Invest. 2013. pii: 67484. doi: 10.1172/JCI67484. 
18. Cedervall J, Zhang Y, Olsson AK. Tumor-Induced NETosis as a Risk Factor for Metastasis and Organ Failure. Cancer Res. 2016;76(15):4311-5. doi:10.1158/0008-5472.CAN-15-3051.

19. Park J, Wysocki RW, Amoozgar Z, Maiorino L, Fein MR, Jorns J, et al. Cancer cells induce metastasissupporting neutrophil extracellular DNA traps. Sci TransI Med. 2016;8(361):361ra138.

20. Kanamaru R, Ohzawa H, Miyato H, Matsumoto S, Haruta H, Kurashina K, et al. Low density neutrophils (LDN) in postoperative abdominal cavity assist the peritoneal recurrence through the production of neutrophil extracellular traps (NETs). Sci Rep. 2018;8(1):632. doi:10.1038/s41598-017-19091-2.

21. Rayes RF, Mouhanna JG, Nicolau I, Bourdeau F, Giannias B, Rousseau S, Quail D, Walsh L, Sangwan V, Bertos N, Cools-Lartigue J, Ferri LE, Spicer JD. Primary tumors induce neutrophil extracellular traps with targetable metastasis promoting effects. JCI Insight. 2019 Jul 25;5(16):e128008. doi: 10.1172/jci.insight.128008. PMID: 31343990; PMCID: PMC6777835.

22. Masucci MT, Minopoli M, Del Vecchio S. Carriero 344 MV The Emerging Role of Neutrophil Extracellular Traps (NETs) in Tumor Progression and Metastasis. Front Immunol. 2020 Sep;16:11:1749.

23. Cedervall J, Dragomir A, Saupe F, Zhang Y, Ärnlöv J, Larsson E, Dimberg A, Larsson A, 475 Olsson AK. Pharmacological targeting of peptidylarginine deiminase 4 prevents cancer-associated kidney injury in mice. Oncoimmunology. 2017 Apr 20;6(8):e1320009.

24. Inoue M, Nakashima R, Enomoto M, Koike Y, Zhao X, Yip K, Huang SH, Waldron JN, Ikura M, Liu FF, Bratman SV. Plasma redox imbalance caused by albumin oxidation promotes lung predominant NETosis and pulmonary cancer metastasis. Nat Commun. 2018 Nov 30;9(1):5116. 480 doi: 10.1038/s41467-018-07550$\mathrm{x}$.

25. Teijeira Á, Garasa S, Gato M, Alfaro C, Migueliz I, Cirella A, de Andrea C, Ochoa MC, Otano I, Etxeberria I, Andueza MP, Nieto CP, Resano L, Azpilikueta A, Allegretti M, de Pizzol M, Ponz-Sarvisé M, Rouzaut A, Sanmamed MF, Schalper K, Carleton M, Mellado M, Rodriguez-Ruiz ME, Berraondo P, Perez-Gracia JL, Melero I. CXCR1 and CXCR2 Chemokine Receptor Agonists Produced by Tumors Induce Neutrophil Extracellular Traps that Interfere with Immune Cytotoxicity. Immunity. 2020 May 19;52(5):856-871.e8. doi: 10.1016/j.immuni.2020.03.001. Epub 2020 Apr 13. PMID: 32289253.

26. Margraf S, Logters T, Reipen J, Altrichter J, Scholz M, Windolf J. Neutrophil-derived circulating free DNA (cfDNA/NETs): a potential prognostic marker for posttraumatic development of inflammatory second hit and sepsis. Shock. 2008;30:352-8. doi:10.1097/SHK.0b013e31816a6bb1.

27. Paunel-Görgülü A, Wacker M, El Aita M, Hassan S, Schlachtenberger G, Deppe A, Choi YH, Kuhn E, Mehler TO, Wahlers T. cfDNA correlates with endothelial damage after cardiac surgery with prolonged cardiopulmonary bypass and amplifies NETosis in an intracellular TLR9-independent manner. Sci Rep. 2017 Dec 12;7(1):17421. doi: 10.1038/s41598-017-17561-1.

28. Vizza E, Corrado G, De Angeli M, Carosi M, Mancini E, Baiocco E, et al. Serum DNA integrity index as a potential molecular biomarker in endometrial cancer. J Exp Clin Cancer Res. 2018, 37(1):16. doi: 10.1186/s13046-018-0688-4. Erratum in: J Exp Clin Cancer Res. 2018, 37(1):35.

29. Rivera-Franco MM, Leon-Rodriguez E, Torres-Ruiz JJ, Gómez-Martín D, Angles-Cano E de la. Luz SevillaGonzález M. Neutrophil Extracellular Traps Associate with Clinical Stages in Breast Cancer. Pathol Oncol Res. 2020 Jul;26(3):1781-1785. doi: 10.1007/s12253-019-00763-5.

30. Zhang Y, Hu Y, Ma C, Sun H, Wei X, Li M, Wei W, Zhang F, Yang F, Wang H, Gu K. Diagnostic. Therapeutic Predictive, and Prognostic Value of Neutrophil Extracellular Traps in Patients With Gastric Adenocarcinoma. 
Front Oncol. 2020 Jun 26;10:1036. doi: 10.3389/fonc.2020.01036. eCollection 2020. PMID: 32714865.

31. Bronkhorst AJ, Ungerer V, Holdenrieder S. The emerging role of cell-free DNA as a molecular marker for cancer management. Biomol Detect Quantif. 2019 Mar 18;17:100087. doi: 10.1016/j.bdq.2019.100087. PMID: 30923679; PMCID: PMC6425120.

32. Sanchez C, Roch B, Mazard T, Blache P, Dache ZAA, Pastor B, Pisareva E, Tanos R, Thierry AR. Circulating nuclear DNA structural features, origins, and complete size profile revealed by fragmentomics. JCI Insight. 2021 Apr 8;6(7):e144561. doi: 10.1172/jci.insight.144561. PMID: 33571170; PMCID: PMC8119211.

33. Lamminaho M, Kujala J, Peltonen H, Tengström M, Kosma VM, Mannermaa A. High Cell-Free DNA Integrity Is Associated with Poor Breast Cancer Survival. Cancers (Basel). 2021 Sep 18;13(18):4679. doi: 10.3390/cancers13184679. PMID: 34572906; PMCID: PMC8467852.

34. Fossati G, Moulding DA, Spiller DG, Moots RJ, White MR, Edwards SW. The mitochondrial network of human neutrophils: role in chemotaxis, phagocytosis, respiratory burst activation, and commitment to apoptosis. $J$ Immunol. 2003;170(4):1964-72.

\section{Figures}

\section{Figure 1}

citH3 staining is present in all endometrial tumor grades. IHC staining of endometrial FFPE tissues with anticitH3 antibody observed by light microscopy, scale 30 um, 20x objective. (A) Staining of citH3 in the leukocyte infiltrate in all EC grades (G1, G2, G3), ranging from low to high signal intensity. (B) Healthy subjects (HSs) are negative for the staining. (C) Box-plots of citH3 staining \% in leucocytes infiltrate in $48 \mathrm{EC}$ samples. Boxes extend from the 25th to 75th percentiles, the horizontal line in the box represent median. (D) Images of G2 and G3 EC tissues (40x objective). Black arrows indicate citH3 release into the extracellular space.

\section{Figure 2}

Confocal microscope imaging of NETosis in EC specimens. (A, E) IF staining of EC tissues of 2 representative patients (G2 and G3) with anti-NE and anti-histone H2B antibodies. Yellow indicates colocalization. (B, F) IF staining with anti-NE antibody and DAPI of the same section of $A$ and $E$ respectively. White indicates colocalization between NE and DNA in the nucleus indicating neutrophils activation. 40x oil objective, confocal single stack. $(C, G) 3 D$ reconstruction of magnifications of regions in white boxes are presented. Merge of 3 fluorocromes are shown. (D) Single staining of panel C detail highlights extracellular extrusion of DNA and citH3. $60 x$ oil objective. $(H, M)$ IF staining with anti-citH3 antibody and DAPI, in tissue from the same 2 patients of panel A and E. 40x oil objective, confocal single stack. ( $, \mathrm{L}, \mathrm{N}, \mathrm{O}$ ) Magnifications of regions in white boxes of figure $\mathrm{H}$ and $\mathrm{M}$. In $\mathrm{L}$ and $\mathrm{O}$ white indicates colocalization between citH3 and DNA in the nucleous, cytoplasm and extracellular space. $60 x$ oil objective. The fine white arrows indicate the presence of colocalization and extracellular extrusion of NE, DNA and citH3. (P) Immunofluorescence images showing different stages of NETosis observed in EC. Left: schematically representation of NETosis stages. Right: NETosis stages found in tissues. Panels I, II, III show resting neutrophils, with trilobed nucleus and intact cytoplasm containing granules 
of NE. In IV, V, VI the nucleus has lost its classic trilobed shape, a yellow color is observed due to the nuclear colocalization of the two markers, testifying the transfer of NE into the nucleus. Panels VII, VIII and IX show the release of neutrophil extracellular traps (NETs).

A

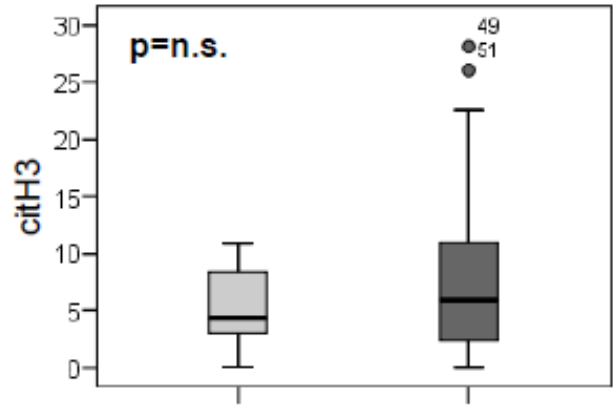

HS

$\mathrm{D}$

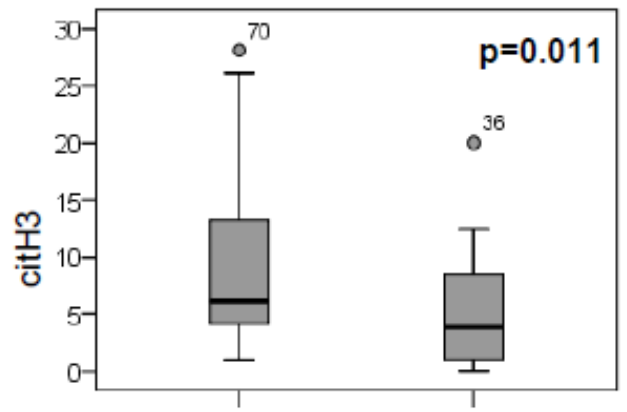

cfmtDNA low cfmtDNA high
B

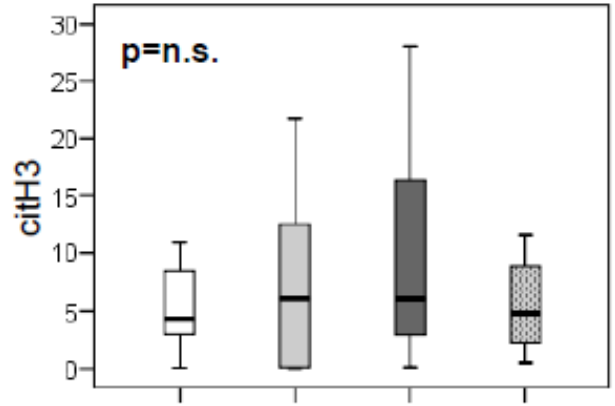

HS G1 G2 G3
$\mathrm{C}$

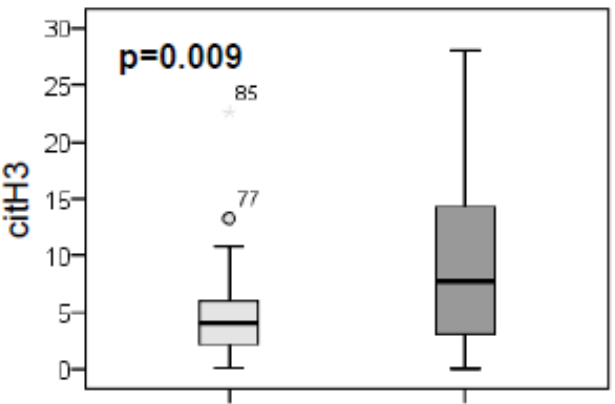

cfDNA low

cfDNA high

E

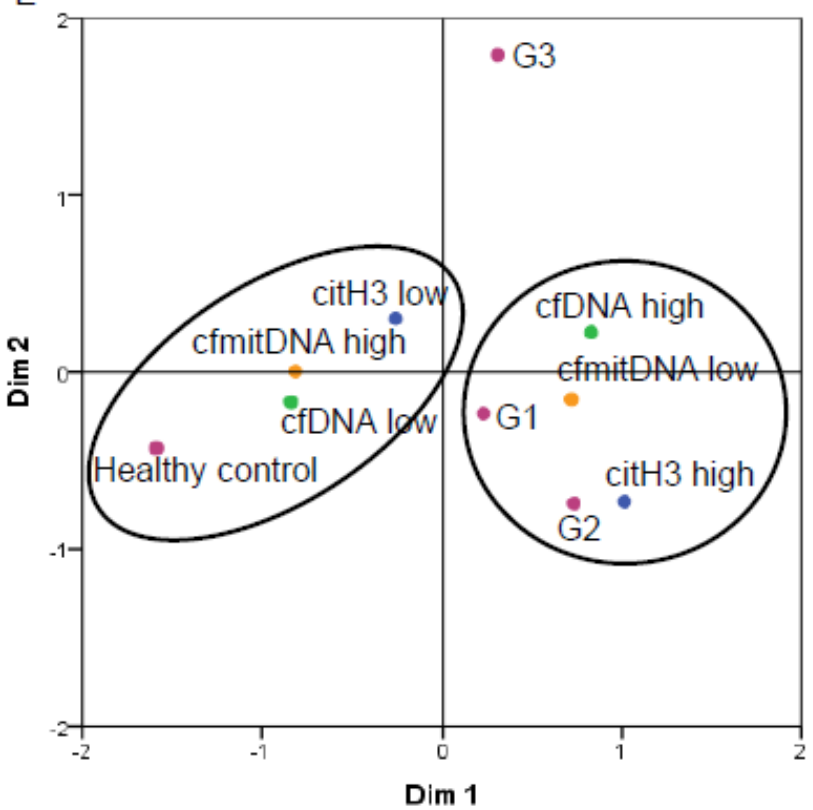

citt3

cfDNA

Grading
cfintDNA

cfintDNA

\section{Figure 3}

citH3 levels are directly related to cfDNA levels and inversely related to cfmitDNA in EC sera. Box-plots of citH3 content in (A) HS and EC sera (B) G1, G2, and G3 EC grades. (C) Cluster analysis of citH3 levels in EC sera displaying a cfDNA content $\leq 712,3$ or $>712$, (D) cfmitDNA content $\leq 16,5$ or $>16,5$. Boxes extend from the 25th to 75th percentiles, the horizontal line in the box represent median. P values, Mann-Whitney test in panel A-C-D; Kruskall-Wallis test in B; n.s.: not significant. (E) Identification of EC-DNA-characteristics related to different grade. Plot of the two-dimension multiple corresponding analysis (MCA) among HSs and EC grades (G1, G2, G3) of cfDNA, cfmitDNA and citH3 categorized according to cut-offs individuated by ROC analysis. 


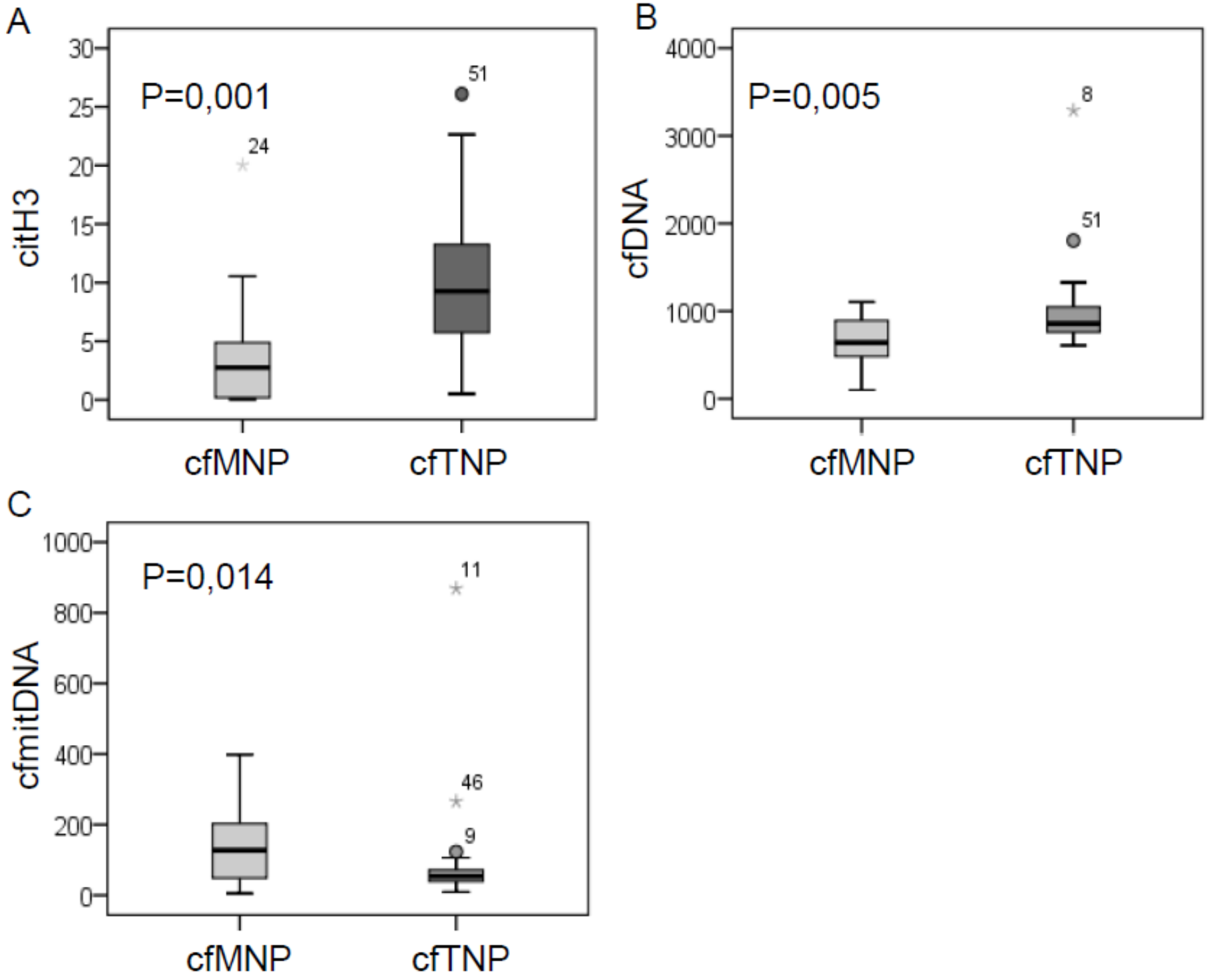

Figure 4

Higher concentrations of citH3 content is associated with the presence of cfTNP in EC sera. Box plot of citH3 (A), cfDNA (B), cfmitDNA (C) levels in the two subgroups of EC serum samples that present cfMNP or cfTNP. The upper border of the box indicates the upper quartile (75th percentile) while the lower border indicates the lower quartile (25th percentile), and the horizontal line in the box the median. P values (Mann-Whitney non-parametric test), n: 19 cfMNP and 25 cfTNP. 

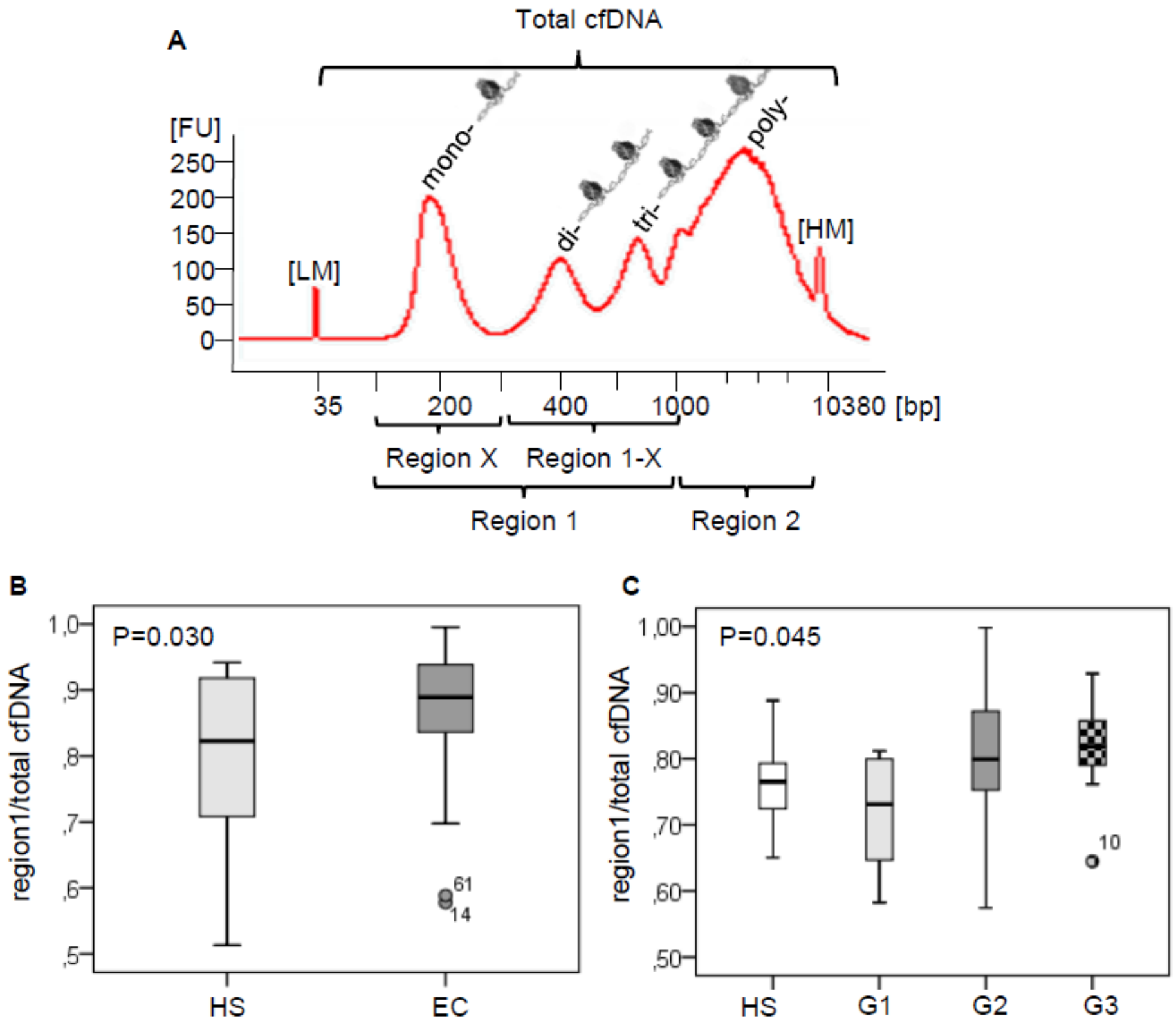

Figure 5

Amount of mono- di- and tri- nucleosome cfDNA related to the total DNA increases in EC serum samples. A) Schematically representation of the different DNA regions analyzed (region X, 1, 1-X, 2, total). B) Box-plots of cDNA region 1 DNA content in HSs and ECs. C) Box-plots of cDNA region 1 DNA content in HSs and in different EC grades (G1, G2, and G3). FU: fluorescence units (proportional to the DNA molarity/concentration), LM: Iow marker, HM: high marker. Boxes extend from the 25th to 75th percentiles, and the horizontal line in the box the median. 
B
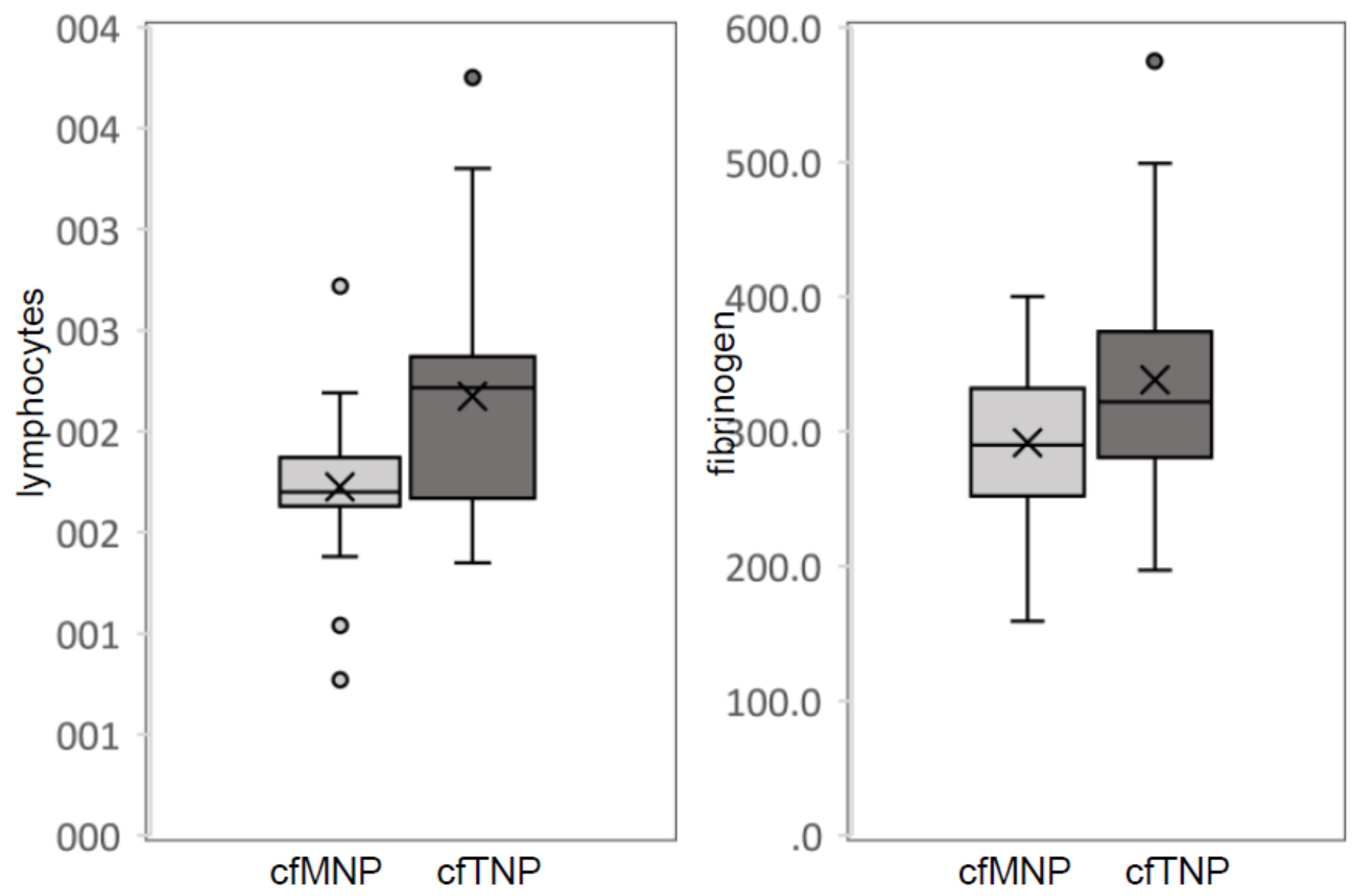

Figure 6

Inflammatory markers increase in the subgroup of cfTNP EC samples. Box plot of lymphocyte (A), and fibrinogen (B) levels in the two subgroups of EC serum samples that present cfMNP or cfTNP cfDNA ladder pattern. Boxes extend from the 25th to 75th percentiles, and the horizontal line in the box the median. n: 19 cfMNP and 25 cfTNP.

\section{Supplementary Files}

This is a list of supplementary files associated with this preprint. Click to download.

- SupplementaryFiguresRonchettietal.pdf

- SupplementaryTableRonchettietal.docx 